EDITORIAL

\title{
Antimicrobial prophylaxis for endocarditis: emotion or science?
}

\author{
H Ashrafian, R G Bogle
}

Heart 2007;93:5-6. doi: 10.1136/hrt.2006.103689

Yet another guideline on the prevention of infective endocarditis has been published, this time limiting prophylaxis to high-risk patients

See end of article for authors' affiliations

.....................

Correspondence to: H Ashrafian, Department of Cardiology, Royal

Brompton and Harefield NHS Trust, Harefield Hospital, Middlesex UB9 6JH, UK; ashrafian@ hotmail.com

Accepted 8 August 2006 Published Online First 16 August 2006
$\mathrm{T}$ he link between dental hygiene and infective endocarditis was made in 1909 by Horder, who observed that "infection is grafted upon a previously sclerosed endocardium...the source of the infecting agent, in most of the cases, is the mouth...". Accordingly, the American Heart Association, European Society of Cardiology and British Cardiac Society published guidelines on the prevention of infective endocarditis. ${ }^{2-4}$ These guidelines recommended administration of antibiotics at the time of dental treatment for all patients deemed to be at a high or moderate risk of infective endocarditis. Recently, new guidelines have been published by the Working Party of the British Society for Antimicrobial Chemotherapy (BSAC). ${ }^{5}$ The BSAC guidelines are important as their recommendations are likely to be adopted by the British National Formulary (BNF) and will be influential in medico-legal disputes. In contrast with previous guidelines, BSAC recommend dental prophylaxis in only three circumstances: previous gically constructed pulmonary shunts or conduits. This radical step to limit prophylaxis to high-risk patients has been welcomed by the dental community who laud the guidelines as "a victory for science and common sense." ${ }^{\prime 6}$

\section{RATIONALE FOR PROPHYLAXIS}

The enduring rationale for prophylaxis has been to identify at-risk patients and protect them during presumptive episodes of increased risk. It is established that dental treatment causes bacteraemia, as does teeth brushing, and also that bacteraemia is associated with seeding of abnormal valves in experimental animal studies. ${ }^{7}$ Traditional guidelines advocating broader prophylaxis were based on extensive animal and human data showing the capacity of dental interventions to induce bacteraemia and that of the antibiotic sulphathiazole to considerably reduce bacteraemia. ${ }^{8}$ Although reducing bacteraemia in higher risk populations may be desirable, the scientific emphasis is moving away from procedure-related bacteraemia towards cumulative bacteraemia. ${ }^{5}$ In actuarial terms, the procedure-related bacteraemia, from activities such as brushing, is estimated to be 6 million times higher than that occurring from a single tooth extraction. Although this infective endocarditis, prosthetic valves, and sur- newer stance is intuitive and influential, it is noteworthy that dental extraction, albeit in children, delivers a larger more prolonged bacteraemia than less vigorous cleaning work. If the risk of infective endocarditis is not just dependant on low-level cumulative exposure but is also influenced by peak sustained intravascular bacterial dynamics, investigators cautiously conclude "prophylaxis [may be] effective and desirable."

The evidence base to which we normally strongly adhere is in this context equally confusing. Anecdotally in England and Wales about 1.35 million dental procedures are carried out on "atrisk patients" (mainly mitral valve prolapse) every year and only about $50 \%$ of these receive appropriate prophylaxis. ${ }^{10}$ Therefore a large number of "at-risk patients" undergo procedures without prophylaxis. Contrast this with only 1500 annual cases of infective endocarditis, most of which are thought to be of non-dental origin. This suggests that for the bulk of patients with conditions such as mitral valve prolapse, prophylaxis is not necessary. Although the literature contains observational studies suggesting the failure of prophylaxis, ${ }^{11-14}$ even these studies indicate a trend towards infective endocarditis in procedures such as extraction and significant dento-gingival manipulation. ${ }^{12}$ Conversely, while there is also evidence that dental work, especially extractions, increase the risk of infective endocarditis fivefold and prophylaxis prevents $70 \%$ of these cases, it is noted that most cases need prophylaxis to prevent a small number of infective endocarditis episodes. ${ }^{15}$ This equipoise has been best articulated by the Cochrane Collaboration who concluded "There is no evidence about whether penicillin prophylaxis is effective or ineffective against infective endocarditis in people at risk who are about to undergo an invasive dental procedure. There is a lack of evidence to support published guidelines in this area and it is not clear whether the potential harms and costs of penicillin administration outweigh any beneficial effect." ${ }^{\prime 16}$

\section{RANDOMISED CONTROLLED TRIAL}

To settle this controversy, many, including the Working Party of the BSAC, have called for a randomised controlled trial to evaluate the risk or benefit of antibiotics. Such a mega-trial would require $>6000$ at-risk participants and would encounter strong ethical concerns as well as being arduous and expensive. ${ }^{1}$ However, there may be

Abbreviations: $A D R$, adverse drug reactions; BSAC, British Society for Antimicrobial Chemotherapy 
another approach; there is an analogy between procedural infective endocarditis and adverse drug reactions (ADR). Both occur rarely, and a randomised controlled trial, unless massive, would be inadequately powered to omit type II errors; both have an unknown and probably heterogeneous temporal association with the inciting event and both affect people who are genetically or structurally predisposed. Unlike ADR, where fiscal incentives and governmental regulation have stimulated pharmacoepidemiology, powered by automated systematic databases, infective endocarditis prophylaxis is hampered by small studies that confuse rather than illuminate. Even the International Collaboration on Endocarditis, boasting a database of $>2200$ patients with infective endocarditis, is probably underpowered to settle this controversy. To settle lingering doubts regarding procedural infective endocarditis, a systematic (inter)national ADR-like reporting system is required which needs to be established between the dental and cardiology communities.

Ultimately, one of the compelling themes of the BSAC guidelines is that prophylaxis is not risk free and is a matter of concern when prescribing treatment to prevent rather than treat disease on the basis of an assessment of actuarial risk or scientific rationale. The pencillins are allergenic and exposure is one of the most common causes of anaphylactic reactions; 1$10 \%$ of patients report a penicillin allergy and the chance of a penicillin reaction is about $5 \%$ for high doses of oral amoxicillin. It has been calculated that in such a large unselected population patient receiving amoxicillin prophylaxis, the risk of death from anaphylactic reactions is five times grater than from contracting infective endocarditis. In addition, minor adverse reactions, unfavourable cost-effectiveness and increasing antibiotic resistance are also reasons cited to avoid antibiotic prophylaxis.

\section{CLINICAL DISCRETION REQUIRED}

The BSAC guidelines are probably an adroit step in the right direction. However, for the overall cohort of patients, prophylaxis may indeed not be appropriate and may indeed be potentially harmful; there are certainly patients with intermediate risks that cohort studies fail to represent. After all, the absence of evidence does not reflect evidence of absence and in such a balanced clinical situation, clinical discretion is of paramount importance. ${ }^{16}$ In the context of this equipoise, such legally compelling guidelines might have done well to have taken a more flexible approach and consulted a broader parish of practitioners that primarily care for and counsel potential and actual patients with infective endocarditis. What then for the cardiologist, dentist and patient? The next few months are likely to be difficult for all. Cardiologists may feel uncomfortable about changing their recommendations for moderate-risk patients in line with the BSAC guidelines, while their own professional societies' published guidelines continue to recommend antibiotics for this group. This is especially true if they perceive the BSAC guidelines to be merely a reassessment of that which was already known. A revised statement of guidance from the British Cardiovascular Society has been helpful and NICE has agreed to undertake an urgent review of antibiotic prophylaxis. ${ }^{18}$ For the dentist the position is also difficult; patients will often have a letter recommending prophylaxis in their dental records or will carry an infective endocarditis dental warning card, whereas these new BSAC guidelines recommend antibiotic prophylaxis in much more limited circumstances. The dentist is an independent clinical practitioner with legal responsibility for prescribing the antibiotics; they cannot simply follow the cardiologist's recommendations blindly. The Chief Dental Officer has written to all dentists with the recommendation that they should adhere to the guidance published in the BNF. If a patient were to have an anaphylactic reaction, the dentist would not be able to adopt the defence that "the cardiologist made me do it". Also for the patient this is a time of uncertainty-particularly for those who have had antibiotic prophylaxis for many years and are now told that it is no longer necessary. Therefore it is essential that patients are adequately counselled on the benefits of good dental hygiene and a discussion is undertaken and documented on the risks or benefits of antibiotic prophylaxis-if that is done hasn't good practice been satisfied?

\section{Authors' affiliations}

H Ashrafian, Department of Cardiology, Royal Brompton and Harefield NHS Trust, Harefield Hospital, Middlesex, UK

R G Bogle, Cardiology and Clinical Pharmacology, Experimental Medicine and Toxicology, Imperial College London, Hammersmith Campus, London, UK

Competing interests: None declared.

\section{REFERENCES}

1 Durack DT. Prevention of infective endocarditis. N Engl J Med 1995;332:38-44

2 Bonow RO, Carabello B, de Leon AC, et al. ACC/AHA Guidelines for the Management of Patients with Valvular Heart Disease. executive summary. A report of the American College of Cardiology/American Heart Association Task Force on Practice Guidelines (Committee on Management of Patients With Valvular Heart Disease). J Heart Valve Dis 1998;7:672-707.

3 Ramsdale DR, Turner-Stokes L. Prophylaxis and treatment of infective endocarditis in adults: a concise guide. Clin Med 2004:4:545-50.

4 Task FM, Horstkotte D, Follath F, et al. Guidelines on Prevention, Diagnosis and Treatment of Infective Endocarditis Executive Summary: The Task Force on Infective Endocarditis of the European Society of Cardiology. Eur Heart $J$ 2004; 25:267-76

5 Gould FK, Elliott TSJ, Foweraker J, et al. Guidelines for the prevention of endocarditis: report of the Working Party of the British Society for Antimicrobial Chemotherapy. J Antimicrob Chemother 2006;57:1035-42.

6 Martin M. A victory for science and common sense. Br Dent J 2006;200:471.

7 Durack DT. Antibiotics for prevention of endocarditis during dentistry: time to scale back? Ann Intern Med 1998;129:829-31.

8 Morris AM, Webb GD. Antibiotics before dental procedures for endocarditis prophylaxis: back to the future. Heart 2001;86:3-4.

9 Lockhart PB, Brennan MT, Kent ML, et al. Impact of amoxicillin prophylaxis on the incidence, nature, and duration of bacteremia in children after intubation and dental procedures. Circulation 2004;109:2878-84.

10 Buckingham JK, Gould UM, Teruitt $G$ et al. Prevention of endocarditis: communcation between doctors and dentists. Br Dent J 1992;172:414-15.

11 Lacassin F, Hoen B, Leport C, et al. Procedures associated with infective endocarditis in adults: a case confrol study. Eur Heart J 1995;16:1968-74.

12 Strom BL, Abrutyn E, Berlin JA, et al. Dental and cardiac risk factors for infective endocarditis: a population-based, case-control study. Ann Intern Med 1998; 129:761-9.

13 van der Meer JT, Thompson J, Valkenburg HA, et al. Epidemiology of bacterial endocarditis in The Netherlands. II. Antecedent procedures and use of prophylaxis. Arch Intern Med 1992;152:1869-73.

14 van der Meer JT, Van Wijk W, Thompson J, et al. Efficacy of antibiotic prophylaxis for prevention of native-valve endocarditis. Lancet 1992:339:135-9.

15 Duval X, Alla F, Hoen B, et al. Estimated risk of endocarditis in adults with predisposing cardiac conditions undergoing dental procedures with or without antibiotic prophylaxis. Clin Infect Dis 2006;42:e102-7.

16 Oliver R, Roberts GJ, Hooper L. Penicillins for the prophylaxis of bacterial endocarditis in dentistry. Cochrane Database Syst Rev, 2004;CD003813.

17 Ramsdale DR, Palmer ND, Fabri BM. Cause for concern? Br Dent J 2004;197:448-9.

18 British Cardiovascular Society. Endocarditis guidelines. http://www.bcs.com/ pages/full_news.asp?NewsID = 17423276 (accessed 13 October 2006). 